\title{
SSI analysis of a massive concrete structure based on a novel convolution/deconvolution technique
}

\author{
J RAJASANKAR*,1 $^{*}$ NAGESH R IYER ${ }^{1}$, B YERRAYA SWAMY ${ }^{1}$, \\ $\mathrm{N}_{\text {GOPALAKRISHNAN }}{ }^{1}$ and P CHELLAPANDI ${ }^{2}$ \\ ${ }^{1}$ Structural Engineering Research Centre, CSIR Campus, Taramani, \\ Chennai 600113 \\ ${ }^{2}$ Indira Gandhi Centre for Atomic Research, Kalpakkam 603102 \\ e-mail: sankar@sercm.org
}

MS received 7 April 2006; revised 6 July 2006

\begin{abstract}
Investigations conducted based on seismic soil-structure interaction analysis of a massive concrete structure supported on a raft foundation are presented in this paper. Linear transient dynamic analysis is carried out using finite element method and imposing transmitting boundary conditions at far field of layered elastic half-space. Analysis is conducted in two phases, namely: (i) free-field analysis of the layered half-space and (ii) seismic analysis of the structure by including soilstructure interaction effects. In the first phase, a simple and novel technique is used to establish free-field excitation at a depth in the half-space. In the second phase, seismic soil-structure interaction analysis of the structure is carried out for the free-field excitation determined in phase-I. Stress resultants experienced by the raft and the stresses at the interface between the rock and raft are evaluated. Critical examination of the results indicates tensile stresses of considerable magnitude at few locations in the rock-raft interface. Typical stress responses at the interface are presented and discussed in the paper.
\end{abstract}

Keywords. Seismic soil-structure interaction; finite element modelling; transmitting boundaries; convolution/deconvolution; transient dynamic response analysis.

\section{Introduction}

Dynamic soil-structure interaction (SSI) is a complex phenomenon for which research suggests solution by using two different methods: sub-structure method and the direct method. In the sub-structure method, the soil-structure system is divided into two sub-structures: (i) super-structure that may include a portion of soil in the neighbourhood and (ii) the remaining unbounded soil. The unbounded half-space is usually represented by simple means using an impedance matrix, which may be directly added to the dynamic stiffness matrix of the

*Corresponding author 
structure. In the direct method, the structure and a fixed extent of half-space are modelled in an identical manner with only distinction in material properties. Sub-structure method operates in frequency domain while the scope of direct method is with time domain. A comprehensive knowledge about sub-structure and direct methods of analysis may be obtained from Kocak \& Mengi 2000, Wegner et al 2005 and Wolf 1985, 1986.

In the numerical analysis of an SSI problem, the main difficulty is in representation of the unbounded half-space on which the structure is supported. Baba (1987) discussed the finite element modelling of the half-space in SSI analysis. Boundary element method (BEM) is also widely used to model the elastic half-space by employing fundamental solutions (Kolar $\&$ Nemec 1989) which implicitly takes care of the geometry of half-space. Such solutions, however, cannot be easily constructed for a layered medium and, therefore, till recently the application of BEM to SSI problems was possible only with severe limitations. Wolf 2003 handled this by introducing scaled boundary finite element method, which combines the advantages of both BEM and finite element method (FEM), for finding efficient solutions. This method provides semi-analytical algorithm which guarantees faster convergence with discretization and also ensures accuracy of the solution. The main advantage of the method is that it does not require a fundamental solution to simulate the geometry effect. It is possible even to couple procedures based on FEM and scaled boundary FEM for three-dimensional dynamic analysis of structures involving unbounded domain (Yan et al 2004). Dutta \& Roy 2002 critically reviewed the alternate analytical and numerical models meant for SSI analysis of structures.

Direct method is computationally amenable to solve an SSI problem in which FEM can be used as a computational platform. In spite of modelling only a limited extent of the halfspace, this method is found to perform well by verifying the results. The effect of semiinfinite foundation half-space is simulated through appropriate boundary conditions called transmitting boundaries (Kocak \& Mengi 2000, Baba 1987) to prevent the reflection of stress waves at the artificially introduced boundaries. Such an approach also suits very well in an implementation using FEM. The purpose of introducing transmitting boundary conditions is to prevent the reflection of stress waves at the artificially introduced boundaries. A better understanding on the performance of the method coupled with the availability of versatile and validated software tools to execute the task is considered as the main advantage for using FEM.

The purpose of this study was to conduct seismic SSI analysis of a large and complex structure supported on a raft foundation. Dimension of the raft which directly rests on the hard rock is $101.8 \mathrm{~m} \times 92.4 \mathrm{~m}$. Analysis was carried out in two phases. In the first phase, free-field analysis of undisturbed elastic half-space described by a volume of $400 \mathrm{~m} \times 400 \mathrm{~m}$ (plan) $\times$ $420 \mathrm{~m}$ (depth) was carried out. The actual layer details of the half-space are represented in the model. Free-field analysis was carried out based on a simple and novel approach for the convolution/deconvolution of excitation. Through this, the free-field excitation at a specified depth in the elastic half-space corresponding to target excitation at ground level was established. Linear transient dynamic analysis of the layered half-space was carried out for this purpose. The exercise was independently carried out for horizontal and vertical excitations. The second phase is meant for evaluating the dynamic response of the structure by using a suitable combined FE model of the structure and the elastic half-space. The extent of the elastic half-space as used in free-field analysis was retained for the second phase except at those zones where it was replaced by the foundation of the structure. FE modelling was thus simplified to a great extent. In generating the FE model of the half-space and the superstructure, the objective of finding only the gross response of the structure was made as the basis to arrive at a coarser mesh. This is also partially driven by the primary goal of this study 
to investigate only overall response and not element level response of the structure. Linear transient dynamic analysis was carried out by subjecting the combined FE model to the freefield excitation determined in the first phase of the analysis. Stress resultants in the concrete raft and the normal and shear stresses developed at the interface between the foundation rock and the raft were evaluated and critically studied.

\section{Transmitting boundary}

Transmitting boundaries effectively eliminate the reflection of stress waves into the foundation due to the artificial boundaries which are introduced solely for the purpose of numerical modelling (Lysmer \& Kuhlemeyer 1969; White et al 1977). In the analysis followed in the present study, at each finite element node on the boundary, the normal and shear forces of viscous (time-dependent) nature were applied to produce the effect of the transmitting boundary conditions. The magnitude of these forces at nodes was due to contribution from neighbouring finite elements. In mathematical terms, the boundary forces were expressed as

$$
\begin{aligned}
& F_{n}=\sigma_{n} \cdot A_{c n}=-\rho c_{d} \frac{\partial u_{n}}{\partial t}, \\
& F_{s}=\sigma_{s} \cdot A_{c s}=-\rho c_{s} \frac{\partial u_{s}}{\partial t},
\end{aligned}
$$

in which $F_{n}$ and $F_{s}$ are forces in normal and tangential directions, $\sigma_{n}$ and $\sigma_{s}$ are normal and shear stresses, $A_{c n}$ and $A_{c s}$ are components of element area in normal and tangential directions, $\rho$ is mass density of material of elastic half-space, $c_{d}$ and $c_{s}$ are dilatational and shear wave speeds in the elastic half-space, and $u_{n}$ and $u_{s}$ are components of particle displacements in normal and tangential directions. Figure 1 shows the forces to be modelled on the transmitting boundaries.

\section{Analysis}

\subsection{Plate resting on elastic homogenous half-space}

Figure 1(a) refers to a $1 \mathrm{~m}$ diameter rigid massless circular plate resting on homogenous elastic half-space subjected to a dynamic sinusoidal point load of unit magnitude. Complex frequency-dependent dynamic impedance functions for the plate response are available as closed-form solutions by following the sub-structure technique (Clough \& Penzien 1993). This benchmark problem has all the essential characteristics of the large structure that is to be analysed except for the size and complexity of the structure, homogenous elastic halfspace and simple excitation. Therefore, before proceeding with an SSI analysis of the massive structure, this plate was analysed to verify the effectiveness of the solution methodology and the finite elements employed in modelling and analysis. Figure 1(b) shows the mathematical model employed for the solution.

Elastic half-space defined by a volume of $5 \mathrm{~m} \times 5 \mathrm{~m} \times 5 \mathrm{~m}$ is included in the discretization. The plate was modelled using four-noded shell elements and eight-noded solid elements were used to model the elastic half-space. Finite element discretization of the rigid plate and the fixed volume of the elastic half-space are shown in figure 2 and the characteristics of the mesh are given in table 1 . The elastic half-space characteristics are listed in table 2. The 


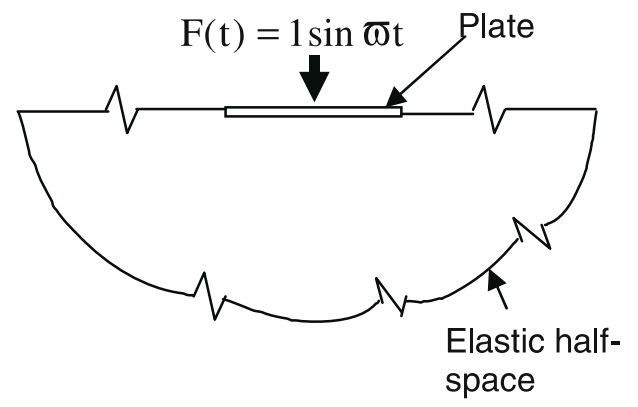

a)

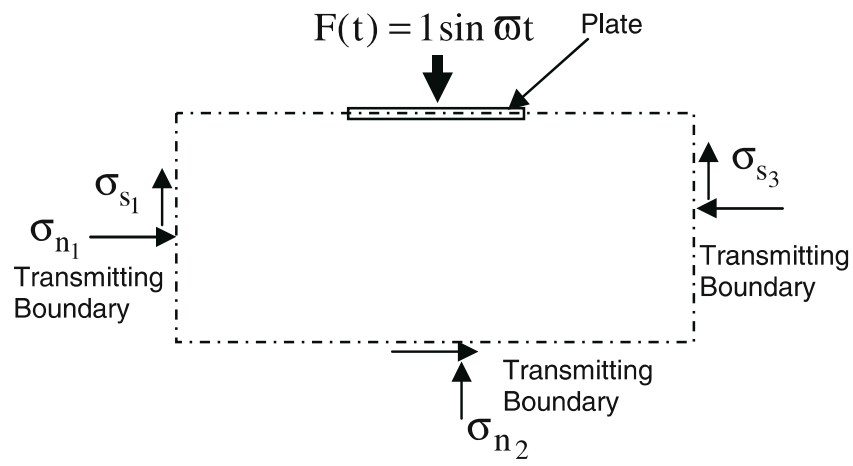

b)
Figure 1. Rigid massless plate resting on elastic half-space (a) Actual problem; (b) Mathematical model.

outer surfaces and the bottom surface of the finite element discretization were imposed with transmitting boundary conditions. The plate was analysed for two different load cases as (i) unit vertical translation load and (ii) unit torsional moment using LS-DYNA software (LSDYNA, 1998). The software has the capabilities required to model and analyse a problem of this nature. Translational load on the plate has been applied all over the plate as uniformly distributed and the torsional moment has been applied as line loads along the perpendicular diameters on the plate.

Dynamic impedance function values have been presented by Clough \& Penzien (1993) for a range of non-dimensional frequency, $a_{o}=R \varpi / c_{s}$, where $R$ is the radius of rigid massless circular plate, $\varpi$ the frequency of excitation, and $c_{s}$ the velocity of shear waves in the foundation medium. Transient dynamic analysis has been carried out to evaluate response

Table 1. Salient characteristics of the finite element mesh.

\begin{tabular}{lcc}
\hline & \multicolumn{2}{c}{ Value } \\
\cline { 2 - 3 } Parameter & Plate & Half-space \\
\hline $\begin{array}{l}\text { No. of elements } \\
\text { Element type }\end{array}$ & 4-noded shell & $\begin{array}{c}1664 \\
\text { 8-noded solid }\end{array}$ \\
\hline
\end{tabular}




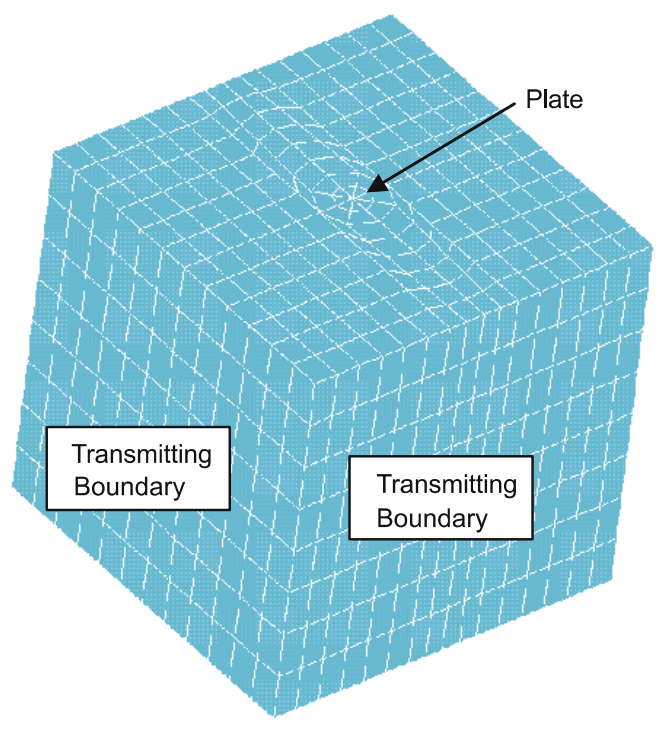

Figure 2. Finite element discretization of circular plate and the half-space.

of the plate for five continuous cycles of loading. The response has been evaluated for a duration of $0.07 \mathrm{~s}, 0.033 \mathrm{~s}, 0.022 \mathrm{~s}, 0.02 \mathrm{~s}, 0.0134 \mathrm{~s}, 0.011 \mathrm{~s}, 0.0095 \mathrm{~s}$, and $0.0084 \mathrm{~s}$ for cases corresponding to non-dimensional frequency $a_{o}=1 \cdot 0,2 \cdot 0,3 \cdot 0,4 \cdot 0,5 \cdot 0,6 \cdot 0,7 \cdot 0$, and 8.0, respectively. The analysis duration was decided to be sufficiently large so as to study the effect of transmitting boundary on results. The analysis was repeated for an idealization including $10 \mathrm{~m} \times 10 \mathrm{~m} \times 10 \mathrm{~m}$ of half-space. It was found that the extent of boundary did not have significant influence on the results since the far-field boundary was modelled to absorb the waves. Further, it was decided that an analysis which includes large volume of elastic half-space is likely to produce only a marginal improvement in the results from which no significant inference can be made to analyse the massive concrete structure. Therefore, results corresponding to only $5 \mathrm{~m} \times 5 \mathrm{~m} \times 5 \mathrm{~m}$ discretization have been presented and instead an effort was made to model and analyse the massive concrete structure. Computed vertical displacements or in-plane rotations at the centre of the plate, as the case may be, have been compared with analytical solution for different non-dimensional frequencies in figures 3(a) and 3(b). The results over the range of frequency values clearly depict the trend of the response and also prove the effectiveness of the solution methodology and the finite elements employed in the analysis.

Table 2. Characteristics of elastic half-space.

\begin{tabular}{ll}
\hline Parameter & \multicolumn{1}{c}{ Value } \\
\hline Density & $1850 \mathrm{~kg} / \mathrm{m}^{3}$ \\
Shear modulus & $1.05 \times 10^{8} \mathrm{~N} / \mathrm{m}^{2}$ \\
Shear wave velocity & $235 \mathrm{~m} / \mathrm{s}$ \\
Young's modulus & $2.72 \times 10^{8} \mathrm{~N} / \mathrm{m}^{2}$ \\
Poisson's ratio & 0.3 \\
\hline
\end{tabular}




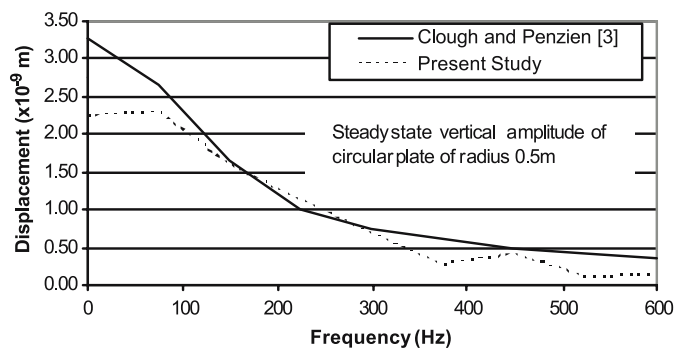

(a)

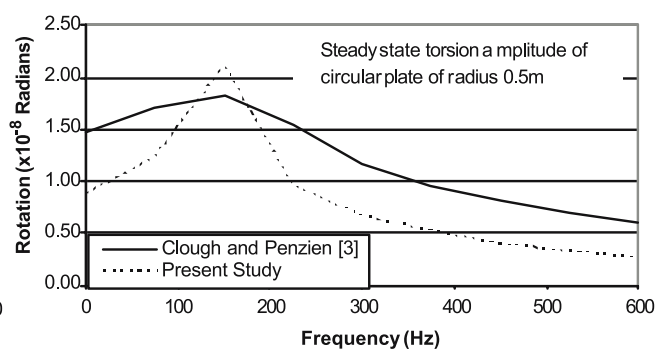

(b)

Figure 3. (a) Comparison of displacement of plate. (b) Comparison of rotation of plate.

\subsection{Massive concrete structure}

The massive concrete structure has a raft foundation to support eight reinforced/pre-stressed concrete buildings. Of these, the tallest building was about $75 \mathrm{~m}$ high. The buildings have different configuration resulting in substantial variation in stiffness and mass distribution in the structure. Apart from this, the sub-surface stratum also has layered formation with significantly different material characteristics that are relevant to decide the seismic response of the structure under interaction conditions. Table 3 gives the extract of the properties of the sub-surface stratum. Target time histories of acceleration in horizontal and vertical directions corresponding to surface level for which response of the structure is to be evaluated are shown in figures 4(a) and 4(b). Acceleration in vertical direction is $2 / 3$ scaled value of that in horizontal direction based on generally accepted practice. The acceleration history represents site-specific synthetic accelerogram generated exclusively for verifying the seismic integrity of the structure.

With these details, seismic SSI analysis of the complex structure was carried out in two phases. The first phase was concerned with finding the free-field excitation at a specified depth in the elastic half-space corresponding to the target excitation (figures 4(a) and 4(b)) at the surface level. In the second phase, dynamic response of the structure is estimated for the free-field excitation found in phase I. For this purpose, linear transient dynamic analysis was carried out by subjecting the combined FE model of the structure and a finite volume of the elastic half-space (as used in phase-I) to the free-field excitation determined in phase-I.

Table 3. Properties of layers in elastic half-space.

\begin{tabular}{|c|c|c|c|c|c|c|c|}
\hline $\begin{array}{r}\text { GL } \\
\text { EL } 280 \mathrm{~m}\end{array}$ & $\begin{array}{l}\mathrm{DBF} / \mathrm{FFL} ; \\
\mathrm{EL} 30.0 \mathrm{~m} \\
\end{array}$ & $\begin{array}{l}\text { Layer } \\
\text { details }\end{array}$ & $\begin{array}{c}\text { Shear wave } \\
\text { velocity } \\
(\mathrm{m} / \mathrm{s})\end{array}$ & $\begin{array}{l}\text { Bulk unit } \\
\text { weight } \\
\left(\mathrm{kN} / \mathrm{m}^{3}\right)\end{array}$ & $\begin{array}{l}\text { Shear } \\
\text { modulus } \\
(\mathrm{MPa})\end{array}$ & $\begin{array}{c}\text { Modulus of } \\
\text { elasticity } \\
\mathrm{E},(\mathrm{MPa})\end{array}$ & $\begin{array}{l}\text { Poisson's } \\
\text { ratio }\end{array}$ \\
\hline \multirow{4}{*}{$\begin{array}{l}\text { EL } 18.0 \mathrm{~m} \\
\text { EL } 11.5 \mathrm{~m}\end{array}$} & & $\begin{array}{l}\text { Dense Sand } \\
\text { Compressible }\end{array}$ & 235 & $18 \cdot 5$ & $102 \cdot 0$ & $286 \cdot 0$ & 0.4 \\
\hline & & $\begin{array}{c}\text { Clay } \\
\text { Weathered }\end{array}$ & 307 & $17 \cdot 0$ & $160 \cdot 0$ & $445 \cdot 0$ & $0 \cdot 39$ \\
\hline & & Rock & 571 & $26 \cdot 0$ & $848 \cdot 0$ & $2187 \cdot 0$ & $0 \cdot 29$ \\
\hline & & Hard Rock & 1390 & $28 \cdot 0$ & $5410 \cdot 0$ & $1.36 \times 10^{4}$ & $0 \cdot 26$ \\
\hline
\end{tabular}



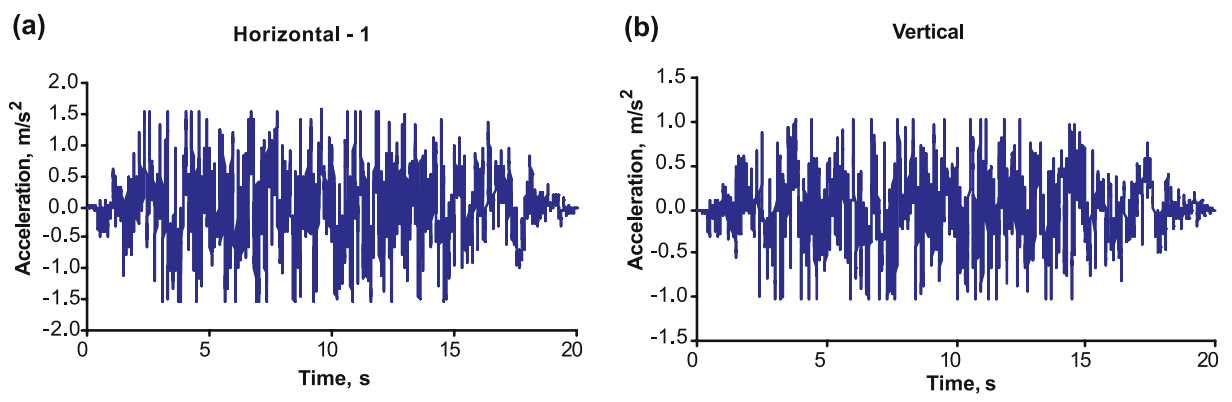

Figure 4. Time-history of acceleration in horizontal and vertical directions. (a) Horizontal direction, (b) Vertical direction.

Thus, even though the two phases of analysis were de-coupled externally, they were linked together in generating input excitation and fixing the extent of the FE model.

3.2a Phase-I: Free-field analysis of the layered half-space: The purpose of conducting free-field analysis was to determine the free-field excitation at a specified depth in the elastic half-space corresponding to a given target excitation at the surface level. This is required to conduct seismic SSI analysis of the structure. In the present study, a simple and novel approach that needs only a couple of linear transient dynamic analyses of the idealized halfspace was followed. Nevertheless, the approach fully complies with the requirement in the linear response regime of the half-space and structure. Basically, it consists of evaluating transfer functions which can be used to transform the input target excitation at surface level to a corresponding one at specified depth of elastic half-space. The transfer functions represent wave propagation characteristics of layered half-space.

As seismic responses are proposed to be evaluated independently to excitations in horizontal and vertical directions, it becomes necessary to evaluate the transfer functions individually corresponding to both the directions. The following steps describe the novel approach followed in Phase-I of the analysis.

(i) An accelerogram with a peak magnitude of $0 \cdot 156 \mathrm{~g}$ and band limited spectrum $(0 \cdot 2 \mathrm{~Hz}$ to $50 \mathrm{~Hz}$ ) was generated for horizontal direction. Similarly, for vertical direction, an accelerogram with a spectrum of $2 / 3$ of the amplitude in horizontal direction was generated. Figures 5(a) and 5(b) show the band limited FFT amplitude spectrum corresponding to accelerogram generated in horizontal and vertical directions.

(ii) An FE model defined by a volume of $420 \mathrm{~m} \times 420 \mathrm{~m}$ (plan) and $400 \mathrm{~m}$ (depth) of layered elastic half-space incorporating non-reflecting boundaries was developed. The plan dimensions denote approximately four times the size of raft. This is shown schematically in figure 6(a). By modelling about 4 times of the base raft dimensions, it is expected that transmitting boundary conditions are imposed on a surface at which approximate plane wave conditions exist. The FE mesh of the half-space is shown in figure 6(b) and the key modelling details are given in table 4 . The control point to which the target excitation corresponds to is also shown in figure 6(b). The FE model includes four different layers of half-space (see details in table 3). The FE model was developed using 8-noded 3-D hexahedron elements. The element is based on fully integrated formulation with three degrees-of-freedom (DOF) per node. Transient dynamic analysis was carried out using the same software as the one used for analysing the plate described 
(a)

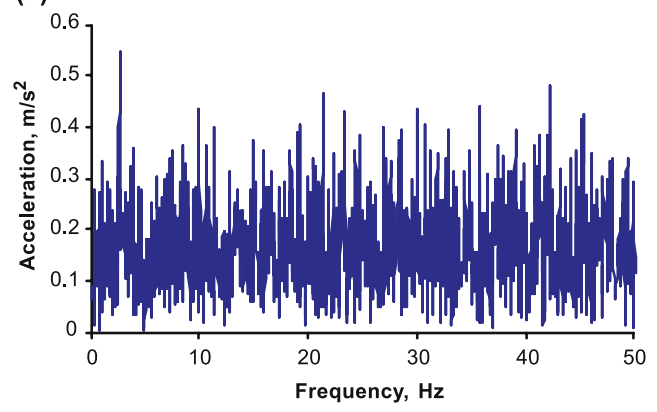

(b)

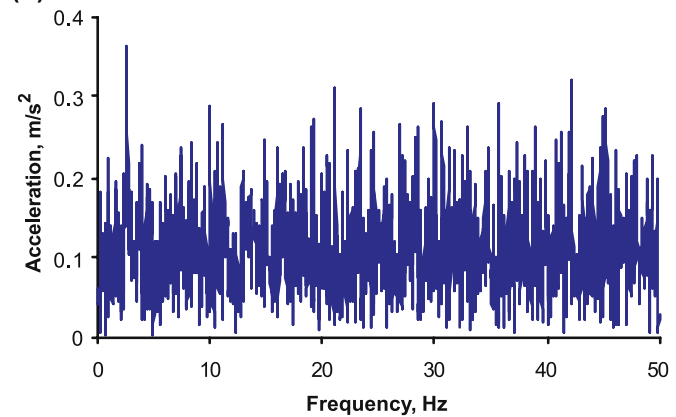

Figure 5. Band limited $(0 \cdot 2-50 \mathrm{~Hz}) \mathrm{FFT}$ amplitude spectrum. (a) Horizontal direction. (b) Vertical direction.

earlier. The layer characteristics available in table 3 were used to develop the material model. The mechanical boundary conditions equivalent to simulate total fixity at the base were imposed for the analysis. Non-reflecting conditions were simulated on the exterior boundaries of the model to prevent stress wave reflections from them into the solution domain. Impedance matching functions based on linear material behaviour were computed for the non-reflecting boundaries and used in the analysis.

(iii) Linear transient dynamic analysis was carried out by subjecting the FE model to base excitation corresponding to the spectrum given in figure 5. A time step of $0.0025 \mathrm{~s}$ was used in the analysis.

(iv) The corresponding time history of acceleration response at a designated control point at $25 \mathrm{~m}$ from outer edge of the model at free surface level was captured.

(v) FFT spectrum of the time histories of acceleration was generated corresponding to horizontal and vertical directions obtained in step (iv).

(vi) The input spectra used in step (i) were scaled with the FFT spectra obtained in step (v) to generate the transfer functions for the layered half-space separately in horizontal and vertical directions.

(vii) The transfer functions generated in step (vi) were used to compute the free-field excitation at $400 \mathrm{~m}$ below the surface level separately for horizontal and vertical directions. Figure 7 gives flow chart describing the sequence of steps followed in phase-I of the analysis.

(viii) The base of the FE model was then subjected to the excitation computed in step (vii) separately in horizontal and vertical directions. The computed acceleration histories at the control point were used to generate the response spectra for horizontal and vertical

Table 4. Details of FE model used for free-field analysis.

\begin{tabular}{lc}
\hline Parameter & Value \\
\hline Nodes & 2704 \\
Elements & 2160 \\
Equations & 7605 \\
\hline
\end{tabular}




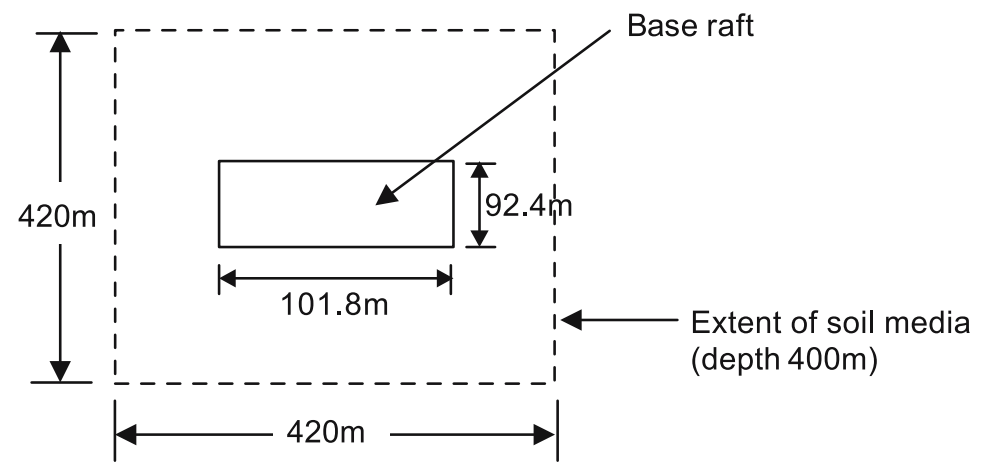

(a)

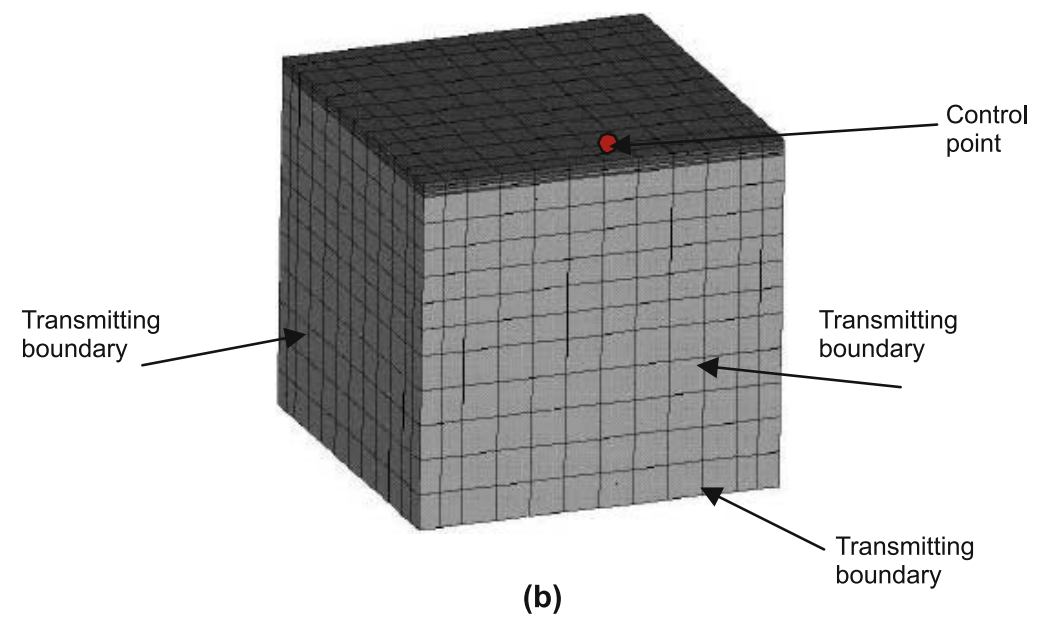

Figure 6. (a) Details of geometry for FE modelling, (b) Finite element model of the half-space for free-field analysis.

directions. Figure 8(a) shows the comparison of response spectra corresponding to target excitation and the response captured at the control point in horizontal direction. Similar comparison for vertical direction is presented in figure 8(b). This clearly shows that the free-field excitation at $400 \mathrm{~m}$ below surface level has been established with reasonable accuracy.

Steps (i) to (vii) described above essentially constitute convolution/deconvolution of excitation. The response spectra of the free-field excitation corresponding to $400 \mathrm{~m}$ below the surface level in horizontal and vertical directions are shown in figures 9(a) and 9(b), respectively.

\subsection{Phase-II: Seismic SSI analysis}

3.3a Finite element model of the structure: As a general concept, the accuracy, validity and convergence of solution depends on the mathematical model conceived for the purpose and the associated FE model employed. In the present study, the finite element model was developed to satisfy the requirements of time domain analysis and the need to accurately represent the stiffness and mass of the structure in the analysis. As explained earlier, the objective 


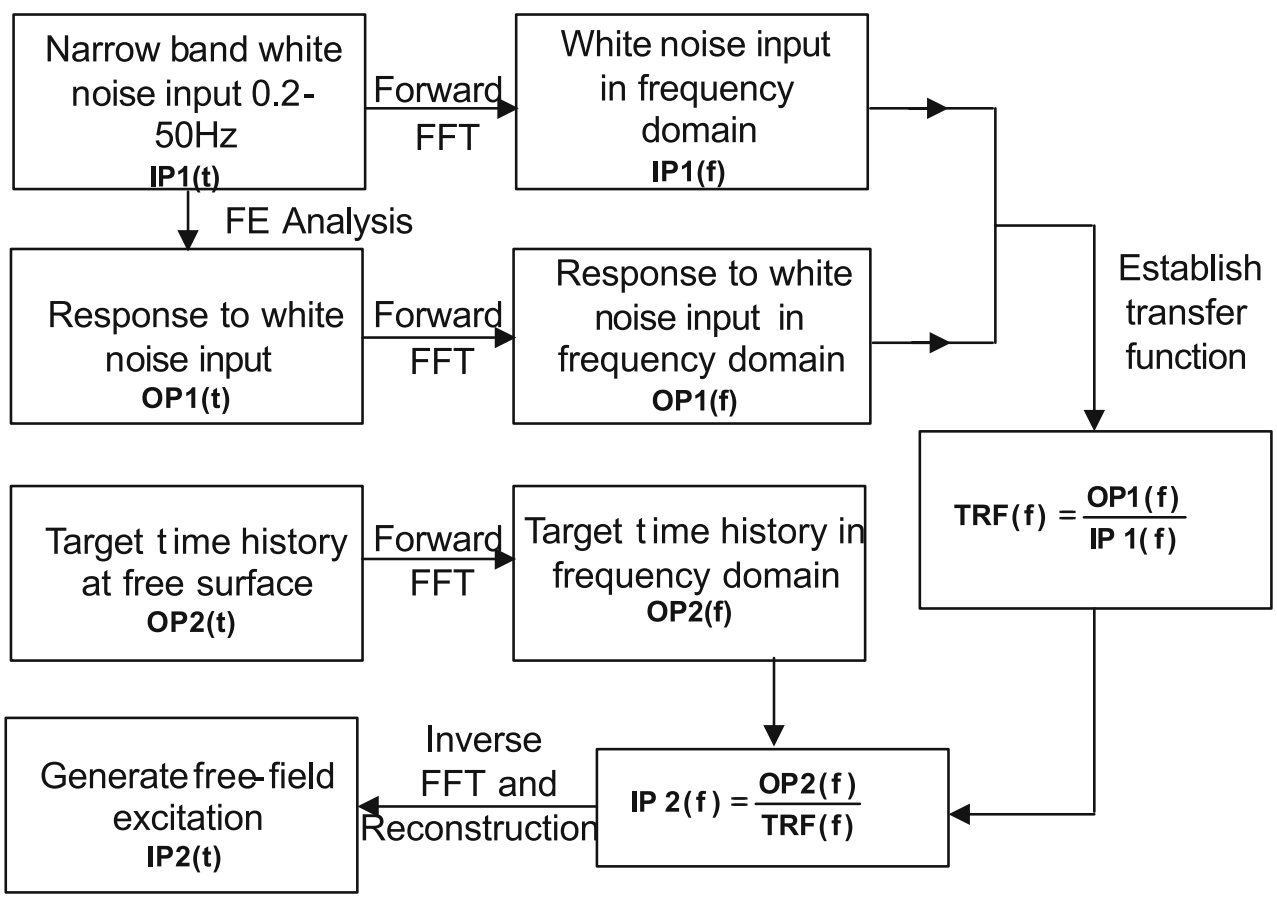

Figure 7. Flow chart of steps followed to establish free-field excitation at $400 \mathrm{~m}$ below ground level.

of present study was to estimate the gross seismic response of the structure. Member level responses are not intended to be evaluated in the investigations. A local analysis using a model that describes the structural characteristics in a detailed manner is essential to study such member level behaviour. Therefore, to achieve modelling and computational efficiency and in conjunction with the objective of the study, a gross model of the structure was developed. During the modelling exercise, wherever possible, equivalent representation of structural elements was followed to minimize the computational demand. Further details regarding the structural modelling are not provided here as it may deviate from the main theme of the investigation.
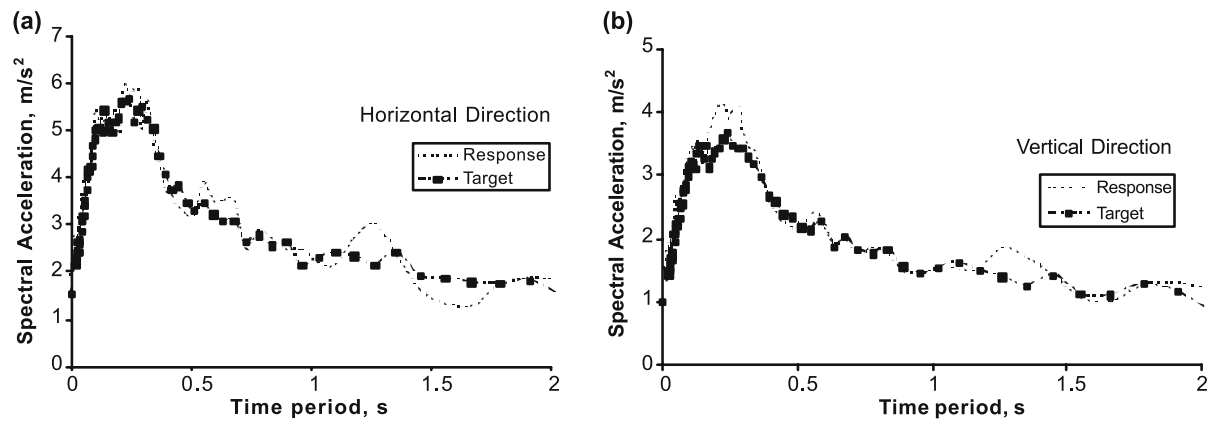

Figure 8. Comparison of response spectra corresponding to target excitation (initial) and the response (final) captured at the control point. (a) Horizontal direction, (b) Vertical direction. 
(a)

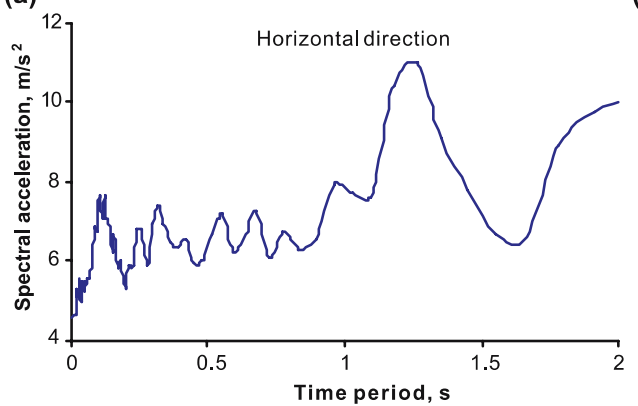

(b)

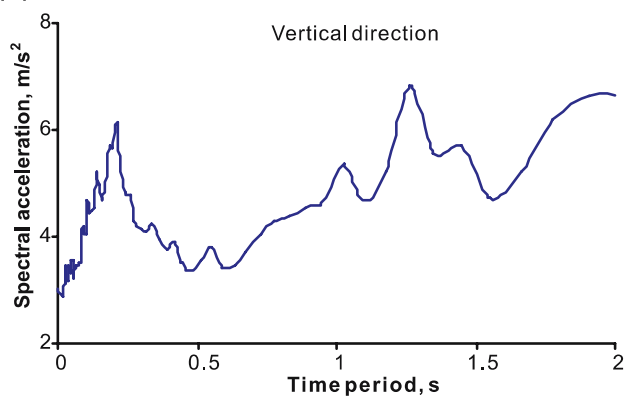

Figure 9. Response spectra of the established free-field excitation. (a) Horizontal direction, (b) Vertical direction.

On deciding at the appropriate modelling strategy and arriving at the necessary structural details, it is the requirement of FE model to depict the necessary structural action through employing suitable finite elements. Grossly, the grading of discretization, choice of finite elements and their sizes are determined to ensure stability in the performance of the model and sufficient accuracy in the results at minimum expense of computational resources. The element sizes are fixed to remain within valid range to capture the results with the anticipated level of detailing. Several alternate models were tried out to arrive at the FE model which is produced in figure 10 whose details are available in table 5(a). The structure was discretized such that the analysis directly produces response at 5854 locations (node points). As table 5(a)

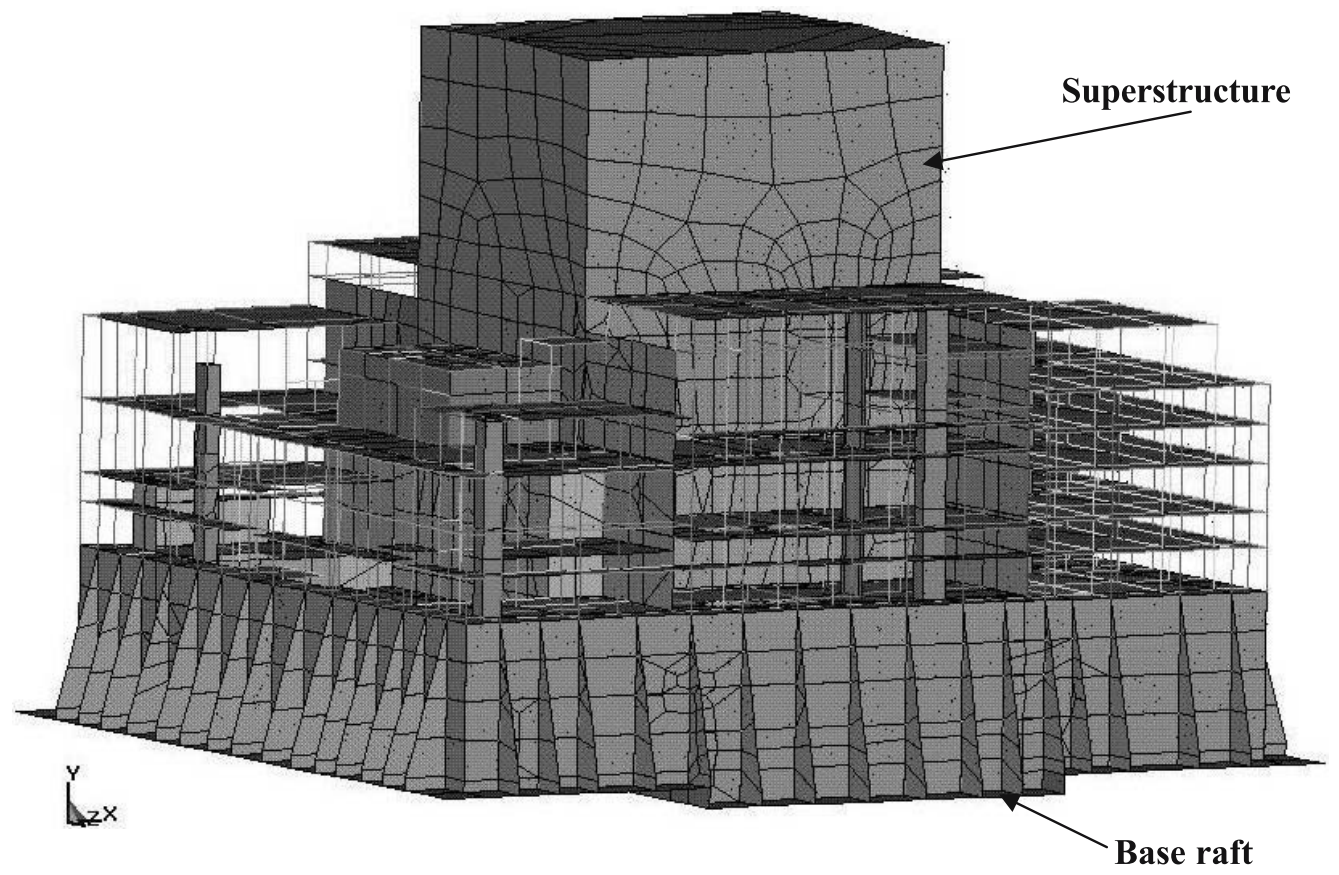

Figure 10. Finite element model of the structure. 
Table 5(a). Details of finite element model of structure.

\begin{tabular}{lc}
\hline Element type & Number of elements \\
\hline Beam & 3440 \\
Shell & 6695 \\
Mass & 1992 \\
\hline
\end{tabular}

shows, the geometry model of the super-structure is restricted to using only $1 \mathrm{D}$ and $2 \mathrm{D}$ finite elements. Foundation raft and the walls of the structure and the buildings were modelled with shell elements.

Further to introducing simplifications in geometry and structural modelling, a similar approach was also followed in material modeling. This exercise is greatly simplified as the interest in the present study is only with linear elastic response of the structure for the chosen seismic excitation. Stiffness modelling was managed through evolving suitable values for elastic modulus of the material. In actual sense, the structure consists of several types of material but they are effectively identified as six distinct material with respect to stiffness representation. The material characteristics are given in table 5(b). Even after having a few material types, the shell elements in the model were assigned with 27 different thicknesses, beam elements were modelled with 77 alternate sections. Detailed manual exercise combined with engineering judgement was carried out to arrive at this geometry and material combinations of the structure with the sole aim of extracting the true dynamic behaviour of the structure. Mass modelling was also paid sufficient attention. The mass of entire super-structure including the base raft is about 2,70,000 tonnes and an approach that suits with the stiffness model was pursued to represent the mass in the model. Mass elements that characterize fixed value of mass at selected nodes of the FE model were followed to distribute the mass of the structure. Free vibration characteristics of the structure were chosen as the criterion to verify the stiffness and mass distribution in the model. Table 5(a) shows that the mass of the structure is lumped at 1992 nodes out of the 5854 nodes in the FE idealization. With all such simplifications in FE modelling, in order to ensure smooth performance of the FE model, the structure was modelled with identical characteristics using ANSYS (2001) and LS-DYNA (1998) software. The basic purpose of conducting this exercise was to make sure that the gross FE model behaves consistently under dynamic loads. At all possible cases, element types of similar characteristics were used in the FE models of both the software. For the LS-DYNA model, 4-

Table 5(b). Material properties of the structural elements.

\begin{tabular}{lcc}
\hline Sl. No. & Elastic modulus (MPa) & Poisson's ratio \\
\hline 1 & $2.7 \times 10^{4}$ & $0 \cdot 2$ \\
2 & $2 \cdot 0 \times 10^{5}$ & $0 \cdot 2$ \\
3 & $1 \cdot 8 \times 10^{5}$ & $0 \cdot 3$ \\
4 & $2 \cdot 0 \times 10^{5}$ & $0 \cdot 3$ \\
5 & $3.4 \times 10^{5}$ & $0 \cdot 2$ \\
6 & $1 \cdot 7 \times 10^{5}$ & $0 \cdot 2$ \\
\hline
\end{tabular}


noded Belytschko-Lin-Tsay shell elements and 2-noded Belytschko-Schwer resultant beam elements were employed. Two point integration order for through-thickness direction in addition to $2 \times 2$ Gauss integration order in the plane of the element was used for the shell element. The element was based on combined co-rotational and velocity-strain formulation. Technically, the computational and performance efficiency of the element is attributed to these two kinematical simplifications. The resultant beam element has 6 DOF per node and at the formulation level itself, a shear factor of $5 / 6$ is included for a $2 \times 2$ Gauss quadrature scheme. In the other case, general shell element (SHELL43 and SHELL63), beam element (BEAM4) and mass element (MASS21) were used to model the structure in ANSYS. Regarding boundary conditions, the base of the structure was approximated to be pinned, i.e., by restraining only the translation DOF.

3.3b Free vibration analysis of structure: As a first step towards understanding the dynamic behaviour of the structure, its free vibration characteristics were evaluated for ideal support conditions. Performance of the FE model was assessed by extracting its free vibration response in the first 40 natural modes. Figure 11 gives comparison of the values of the natural frequencies computed using both models for the initial twenty modes of vibration. The figure indicates a general agreement with only minor difference in a consistent manner which can be attributed to the difference in the behaviour of finite elements used in the model. Mainly the performance of the model over a large frequency range is ensured by studying the results of free vibration analysis.

3.3c Extended finite element model of structure and elastic half-space: For conducting SSI analysis, an extended FE model of the structure and its neighbourhood half-space was developed. The same extent of half-space as modelled for the free-field analysis was included in the extended FE model. The raft foundation of the structure directly rests on the hard rock,

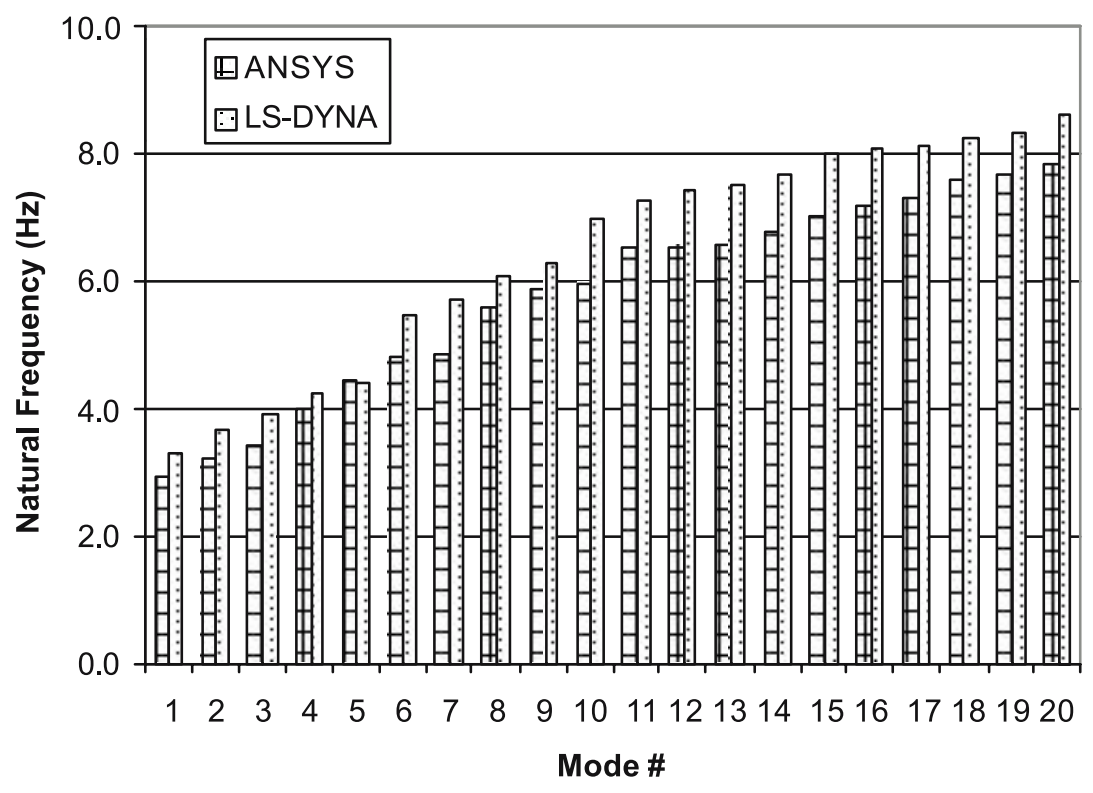

Figure 11. Natural frequencies (in $\mathrm{Hz}$ ) of the super-structure. 
i.e., at 11.5 below the ground level. In one sense, an equal volume of half-space was replaced by a super-structure in the extended FE model compared to the one used in free-field analysis. In doing so, exactly the same FE mesh was imposed on the base raft and the top surface of the half-space in contact with it to satisfy compatibility requirements. Similar aspects were taken care of in developing FE meshes for the walls of super-structure by satisfying with the FE mesh of the surrounding confining soil. Extra care was taken to develop a properly graded FE discretization by establishing compatible meshes between adjacent regions. Considering the extreme difficulty to satisfy this criterion along the walls and counterforts, which has severely unstructured mesh, the condition of perfect matching of meshes was relaxed at necessary locations so that the counterforts were modelled to have contact only with walls and allowed to act independent of the confining soil. However, confining soil is modelled to have perfect contact with the walls. Even from the practical considerations, this is an acceptable relaxation as the structure is likely to move independent of the confining soil.

A large portion of the half-space included in the model was discretized with 8-noded solid hexahedron finite elements. Considerable difficulty was encountered while generating FE mesh for the half-space adjacent to the raft as well as adjacent to the wall. In addition, the complex geometry of the junction between wall, counterfort and raft coupled with varying thickness of the foundation layers posed difficulties to develop a mesh by using only hexahedron elements. In view of this, the region in the neighbourhood of the structure was meshed with 4-noded tetrahedron and 5-noded pentahedron solid elements. By this way, extreme care was taken to ensure absolute compatibility of mesh while developing the model. These elements also, however, belong to the same family of 8-noded hexahedron elements and, therefore, are expected to perform with matching accuracy but with slightly more demand on the computational resources. Different views of the extended FE model are shown in figures 12(a), 12(b) and 12(c). Table 6 presents the important characteristics of the extended FE model.

3.3d Seismic SSI analysis: Seismic response of the structure was evaluated by considering the SSI effects. Linear transient dynamic analysis was carried out by subjecting the extended FE model to the free-field excitation evaluated in phase-I. Apart from the seismic excitation, only the self weight of the super-structure was included in the analysis. As the structure is unsymmetric in plan, two independent analyses were carried out for excitations along the horizontal directions. In addition, an analysis was carried out for excitation in vertical direction. The responses of the structure for the entire duration of $20 \mathrm{~s}$ of excitation were computed at a constant sampling interval of $0.01 \mathrm{~s}$. As explained earlier, the primary objective of the present analysis was to estimate the overall response of the structure and not member level forces. The structure and the support conditions are represented in accordance with this objective and the stress resultants comprising of moments and shear forces experienced by the base raft and vertical separation stresses developed at the interface were captured and studied.

\section{Results and discussions}

\subsection{Bending moment and shear forces in raft}

Variation of the bending moment and shear forces during the entire duration of analysis was studied to identify the critical values of these quantities. Animated contours of the response quantities provided a better perspective about their variation. Figures 13(a) and 13(b) show typical variation of shear force and bending moment at an instant during excitation. The main observation is maximum moment experienced by considerable area of the base raft is about 

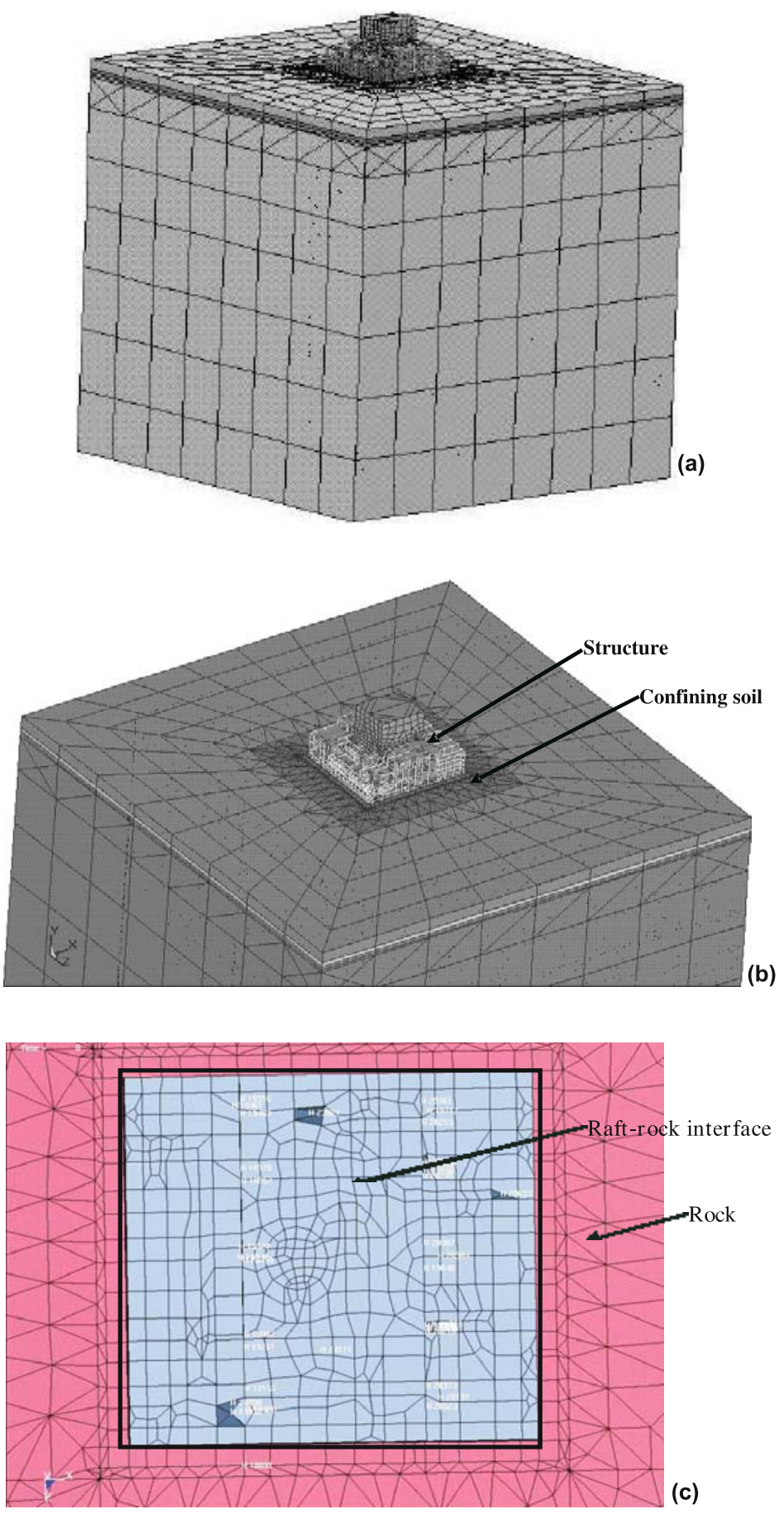

Figure 12. (a) Extended FE model, (b) Zoomed view of extended FE model, (c) Zoomed view of FE discretization at interface between base raft and half-space. 
Table 6. Salient characteristics of the extended FE model.

\begin{tabular}{lllc}
\hline \multicolumn{2}{c}{ FE Model details } & Soil/structure & Critical damping (\%) \\
\hline Solid elements & 14269 & Hard rock & 0.5 \\
Beam elements & 3440 & Weathered rock & 1 \\
Shell elements & 7772 & Clay & 2 \\
Nodes & 9580 & Dense sand & 3 \\
Equations & 46011 & Structural elements & 7 \\
Mass modelled in the & 270631 & & \\
$\quad$ super-structure & tonnes & & \\
Duration of analysis & $20 \mathrm{~s}$ & & \\
Time step & $0.01 \mathrm{~s}$ & & \\
\hline
\end{tabular}

$500 \mathrm{kNm} / \mathrm{m}$ while only a few isolated zones experience bending moment of the order of $600 \mathrm{kNm} / \mathrm{m}$. It is considered prima-facie that the higher values in isolated zones could be due to the modelling strategy followed in the analysis coupled with numerical disturbances and, therefore, has to be interpreted in proper perspective. Moreover, a similar trend was observed in all the three analyses carried out which further confirmed the above proposition.

Shear forces experienced by the raft in both tangential and normal directions were studied to find out the critical values. Among the three analysis carried out, as expected, it is seen that excitation along vertical direction produce maximum shear in the raft. Maximum shear experienced by raft is about $920 \mathrm{kN}$. This value is again confined to a smaller zone of the raft while the maximum value of the response experienced by reasonably large area of the raft in concurrence with the finite element discretization followed can be taken as $400 \mathrm{kN}$.

\subsection{Stresses at interface between raft and foundation}

One of the possible modes of response of large structure resting on a rock foundation is by sliding or rocking which in extreme case could result in overturning. As the raft and the foundation may respond independently to the random seismic excitation, there is a possibility for them to get separated even partly during discrete time intervals within the duration of excitation. A rigorous analysis by invoking contact/separation algorithm is most ideal to handle such conditions. Another indirect and simple way, however, to handle this condition is to verify the magnitude of the uplift stresses/forces developed at the interface and make

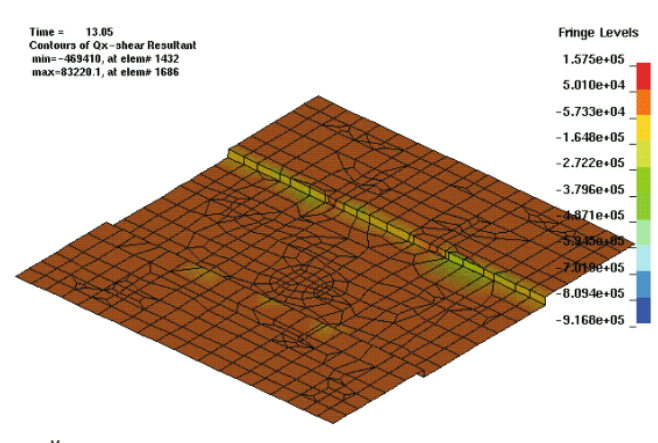

(a)

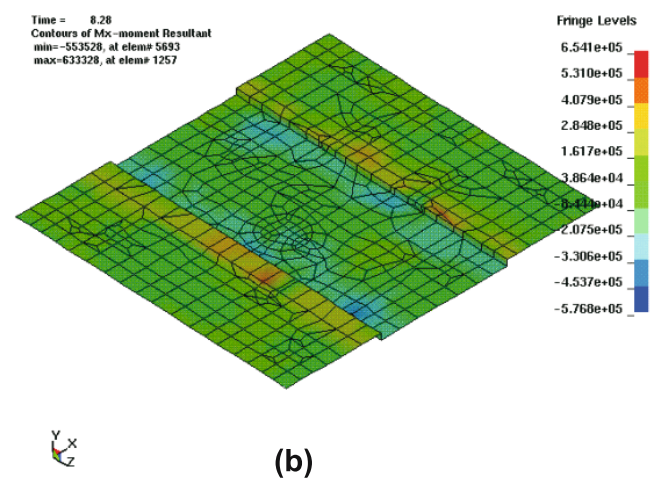

(b)

Figure 13. Shear force and bending moment distribution in raft. (a) Shear force, (b) Bending moment. 
additional structural provisions to eliminate the possibility of lifting. Thus a check on the magnitude of stresses developed at the interface will provide an indication about the type of precautions to be taken or structural modifications to be carried out to ensure continuous connectivity between the foundation raft and rock. While the lateral force generated due to base excitation provide the overturning moment, the self-weight of the structure contribute to the restoring moment. A global analysis like the one carried out here will provide enough clues to assess the possibility of uplifting or overturning. Therefore, the contour of vertical component of stresses developed at the interface between the raft and foundation is closely watched to critically infer its behaviour. The contours of positive (tensile) stresses at two selected instances of time are captured and shown in figure 14. The values in the contour plots indicate tensile stresses of considerable magnitude in the interface. Another typical observation from the stress contours is that the regions experiencing tensile stresses were confined to isolated zones similar to that noticed for bending moment. These zones are, however, found to experience tensile stresses continuously throughout the entire duration of excitation. Similar to the observation in bending moment, the isolated occurrence of critical values is attributable partly to the stiffness and mass idealisations followed in the analysis. Therefore, a knowledgeable interpretation of the results in adherence with the model adopted is necessary to derive valuable conclusions from the analysis. Four locations at the interface marked with circles experience a maximum vertical stress of about $500 \mathrm{kN} / \mathrm{m}^{2}$. The remaining regions, however, show only either nominal tensile stresses or compressive stresses. Apart from this, exactly opposite edges of the raft is found to exhibit lifting and resting (rocking action) in a cyclic manner which is typical of the response of a structure in a earthquake type loading. Such a response at a particular instant is shown in figure 15, which further indicates that the magnitude of vertical stresses at the edges are less compared to that experienced in

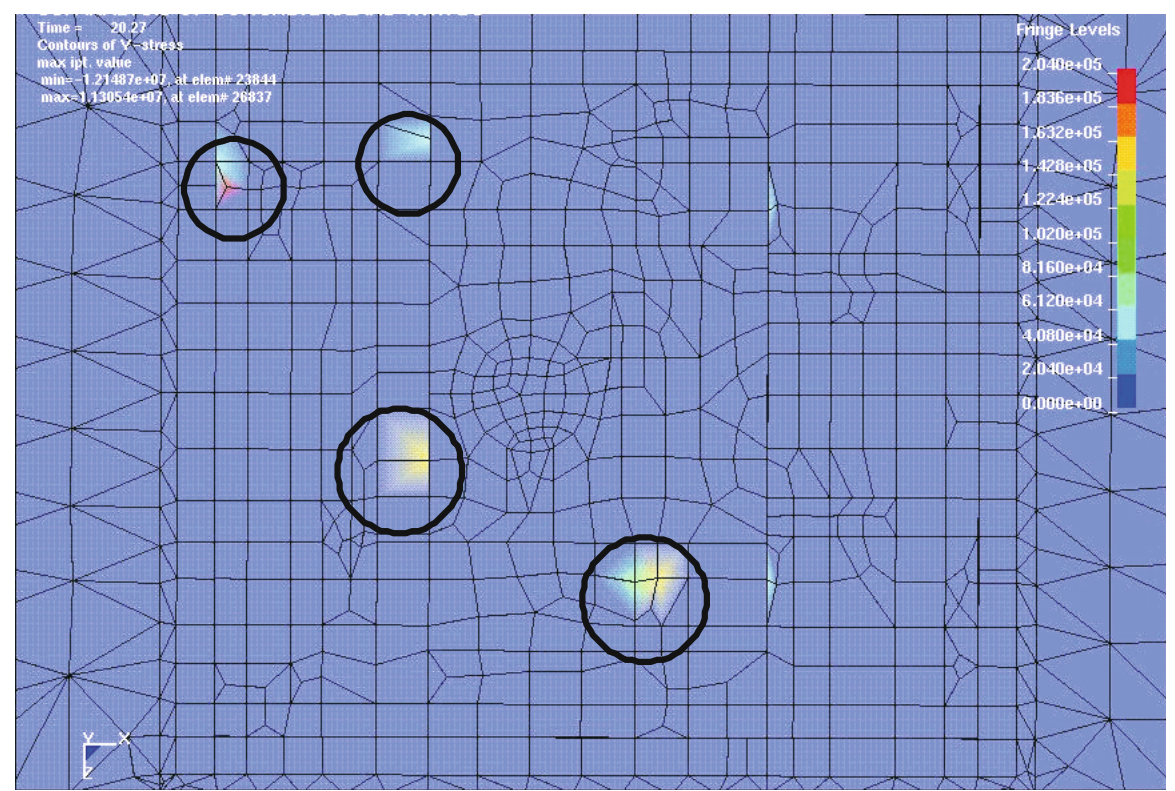

Figure 14. Contour of vertical stresses at the interface between foundation rock and raft for H1 excitation at time $t=18.77 \mathrm{~s}$. 


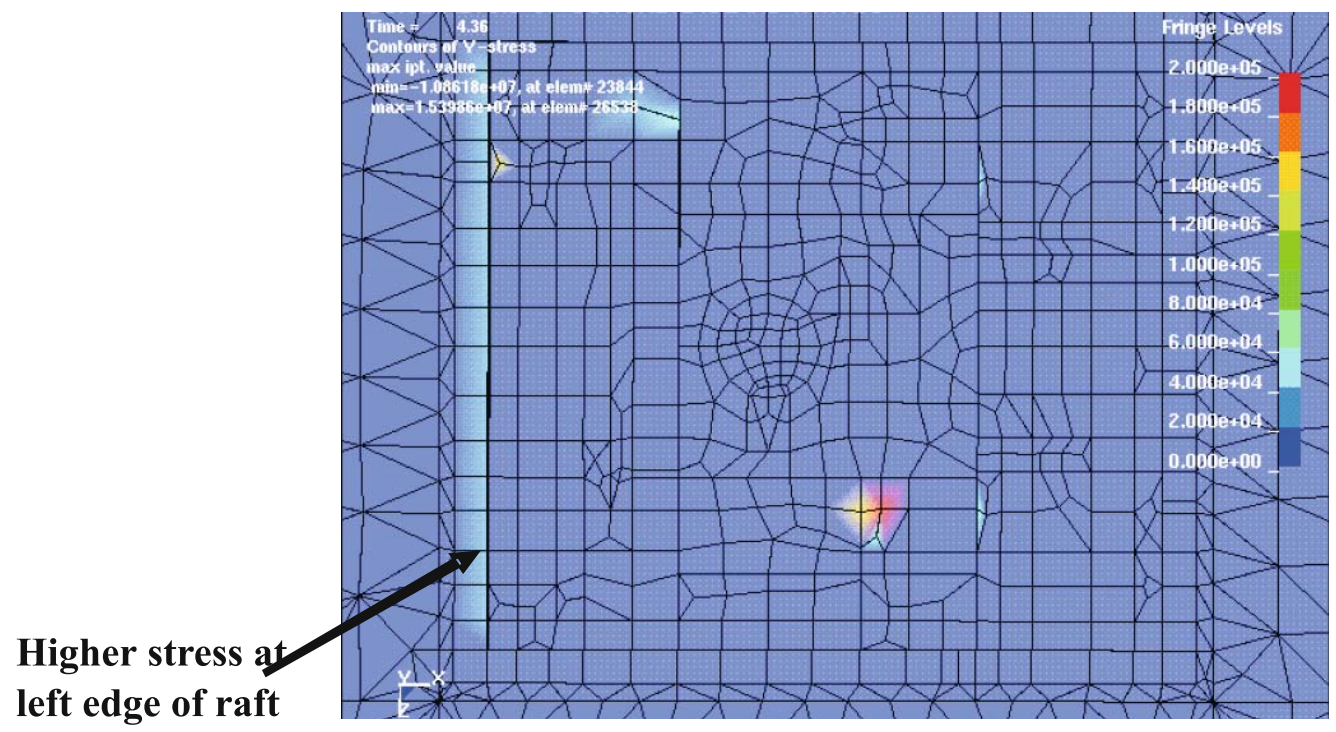

Figure 15. Stress contour indicating lifting-up of left edge of the raft.

patches in the interior of the raft. This particular trend in the computed response which can be easily explained based on the physical behaviour of a structure like the one being analysed provide enough evidence to conclude the correctness of the modelling approach followed and the validity of the results obtained. Among the two horizontal excitations, the one along the longer span of the raft is found to be critical.

\section{Summary and concluding remarks}

Seismic soil-structure interaction (SSI) analysis of a large structure resting on layered elastic half-space is presented in this paper. The aim of the analysis is to find an overall response of the structure in an earthquake excitation. The paper presents a brief theoretical background on modelling a problem that involves dynamic SSI effects and subsequent issues to be addressed in the analysis. Seismic SSI analysis was carried out using FEM and the principles of linear transient dynamic analysis. For this purpose, an FE model of the super-structure including the raft and a finite volume of layered half-space was developed. To fulfil the aim of the analysis envisaged, a grossly simplified FE model of the super-structure is developed. Equivalent structural element properties both in geometry and material aspects were arrived at to model the structure in FE analysis.

Before carrying out the SSI analysis of the massive structure, similar analysis was carried out on a rigid maseless circular plate resting on homogenous elastic half-space. This problem also involves all the essential features of a typical SSI analysis but in a much smaller and simpler scale. In addition, analytical solution is available in literature by virtue of its simple geometry and well-defined loading. The computed displacements and rotations of the plate over a suitable range of excitation frequencies are found to show the trend indicated by the analytical solution. The exercise proved to be useful to study the intricacies in modelling a SSI problem. 
Seismic SSI analysis of the massive structure was carried out in two phases, namely, (i) freefield analysis of the layered elastic half-space and (ii) SSI analysis of the structure. In the first phase, free-field analysis of the layered half-space was conducted to establish the free-field excitation at $400 \mathrm{~m}$ below the ground level that was to be used in the second phase of the analysis. This exercise, termed conventionally as convolution/deconvolution of excitation, was carried out by a novel technique involving a couple of linear transient dynamic analysis and to derive the transfer functions to characterize wave propagation in the layered medium. This was carried out separately for excitation along horizontal and vertical directions. To carry out the second phase of analysis, a gross FE model of the structure was built with usual approximations allowed in a global analysis. Using this model, for ideal boundary conditions, free vibration analysis of the super-structure was carried out to extract its natural frequencies. Duplicate analysis was carried out using ANSYS and LS-DYNA software to understand and assess the implications of the simplified modelling strategy and to ensure the stability in the performance of the model. The computed free vibration responses matched reasonably well to prove the adequacy and performance of the model. Then an extended FE model of the structure by including the same finite volume of half-space as used in the freefield analysis was developed to carry out SSI analysis. The artificial boundaries introduced to limit the elastic half-space in the model were assigned the property to absorb the stress waves emanating from the interface with the structure during an earthquake excitation. Linear transient dynamic analysis was carried out by applying the free-field excitation derived in the first phase of the analysis. Three independent analyses were carried out corresponding to three orthogonal directions for a duration of $20 \mathrm{~s}$.

Time histories of shear forces and bending moments in the concrete raft were evaluated. Apart from this, the vertical stresses developed at the interface between the concrete raft and foundation rock was closely scrutinized. Contours of such stress values gave clear indication on the possibility of separation of the concrete raft from the foundation rock. Both the stress resultants in the raft and the vertical stresses at the interface showed a particular trend of patch-wise higher values compared to a large region of lesser values. This is partly attributable to the approximation introduced in modelling the stiffness and mass of the structure. Among the excitations, the one along the longer span of the super-structure was found to be critical. This again was confirmed by closely following the time variation of stresses which showed tensile values at the edges of the raft intermittently in the duration of analysis. As a whole, the investigations provided worthwhile information about seismic integrity of the structure in an anticipated seismic excitation.

The authors express their thanks to Dr N Lakshmanan, Director, SERC for guidance, keen interest and participation in the discussions during every phase of the investigations. The constructive discussions with our colleagues Dr G S Palani and Shri S Bhaskar and Shri A Rama Chandra Murthy on this topic are appreciated. This paper is being published with the kind permission of the Director, SERC.

\section{List of symbols}

$F_{n}, F_{s}$ forces in normal and tangential directions, $\mathrm{N}$;

$\sigma_{n}, \sigma_{s} \quad$ normal and shear stresses, $\mathrm{N} / \mathrm{m}^{2}$; 
$A_{c n}, A_{c s} \quad$ components of element area in normal and tangential directions, $\mathrm{m}^{2}$;

$\rho \quad$ mass density of material of elastic half-space, $\mathrm{kg} / \mathrm{m}^{3}$;

$c_{d}, c_{s} \quad$ dilatational and shear wave speeds in the elastic half-space, $\mathrm{m} / \mathrm{s}$;

$u_{n}, u_{s} \quad$ components of particle displacements in normal and tangential directions, $\mathrm{m}$;

$a_{o} \quad$ non-dimensional frequency;

$R \quad$ radius of rigid massless circular plate, $\mathrm{m}$;

$\varpi \quad$ excitation frequency, $\mathrm{rad} / \mathrm{sec}$.

\section{References}

ANSYS Ver 6.0 2001 Theory Manual, ANSYS Inc., (USA: Pennysylvania)

Baba K 1987 Dynamical behaviour of the soil-foundation interaction system due to earthquake type loading, in soil-structure interaction (Ed.) A S Cakmak, (Amsterdam: Elsevier Science Publishers B.V.)

Clough R W, Penzien J 1993 Dynamics of structures, Edn. 2, (USA: McGraw Hill Company Inc.)

Dutta S C, Roy R 2002 A critical review on idealisation and modelling for interaction among soilfoundation-structure system. Comput. Struct. 80: 1579-1594

Kocak S, Mengi Y 2000 A simple soil-structure interaction model. Appl. Math. Model. 24: 607-635

Kolar V, Nemec I 1989 Modelling of soil-structure interaction, (New York, USA: Elsevier Science Publishing Co. Inc.)

LS-DYNA Ver. 9701998 Theoretical Manual, Livermore Software Technology Corporation, (USA: California)

Lysmer J, Kuhlemeyer R L 1969 Finite dynamic model for infinite media. J. Eng. Mech. 95: 859-877

Wegner J L, Yao M M, Zhang X 2005 Dynamic wave-soil-structure interaction analysis in the time domain. Comput. Struct. 83: 2206-2214

White W, Valliapan S, Lee K 1977 Unified boundary for finite dynamic models. J. Eng. Mech. 103: 949-964

Wolf J P 1985 Dynamic soil-structure interaction, (New Jersey: Prentice-Hall Inc.)

Wolf J P 1986 A comparison of time domain transmitting boundaries. Earthquake Eng. Struct. Dyn. 14: 655-673

Wolf J P 2003 The scaled boundary finite element method, John Wiley Sons, (England: Chichester)

Yan J, Zhang C, Jin F 2004 A coupling procedure of FE and SBFE for soil-structure interaction in the time domain. Int. J. Numer. Methods Eng. 59: 1453-1471 